Original Paper http://ajol.info/index.php/ijbcs http://indexmedicus.afro.who.int

\title{
Effet du temps de chauffage des graines de palmier à huile (Elaeis guineensis Jacq.) sur quelques métabolites au cours du processus de la germination
}

\author{
Martine Manéhonon BEUGRÉ ${ }^{1 *}$, François Kouakou Yao KONAN ${ }^{1}$, \\ Salomé Edwige Sopie YAPO ${ }^{1}$, Eugène Kouakou KONAN ${ }^{2}$ et Justin Yatty KOUADIO ${ }^{1}$ \\ ${ }^{1}$ Université Jean Lorougnon Guédé, UFR Agroforesterie, BP 150 Daloa, Côte d'Ivoire. \\ ${ }^{2}$ Programme Palmier, Laboratoire de culture in vitro, Centre National de Recherche Agronomique (CNRA) \\ Station la Mé, 13 BP 989 Abidjan 13, la Mé, Côte d'Ivoire. \\ *Auteur correspondant ; E-mail : beugremartine@yahoo.fr
}

\section{REMERCIEMENTS}

Les auteurs remercient Winrock International pour sa contribution financière à la réalisation de ce travail.

\section{RESUME}

Pour une bonne disponibilité des graines germées sélectionnées et dans un court délai, une technique de "germination par chaleur sèche" modifiée a été initiée. À cet effet les graines ont été traitées à différentes durées de chauffage. Les dosages des polyphénoloxydases (PPO), de la phénylalanine ammonia-lyase (PAL), des composés phénoliques totaux et des protéines ont été réalisés pour 40,60 et 80 jours à $40{ }^{\circ} \mathrm{C}$. Les résultats obtenus au niveau des albumens sont négligeables. Chez les embryons, les activités enzymatiques et la teneur des protéines ont diminué avec l'augmentation de la durée de chauffage avec respectivement de 177,7 à 35,07 DO/min/g M.S pour les PPO, de 928,54 à $334 \mathrm{mM}$ d'acide cinnamique/min/g M.S pour la PAL et de 92,4 à $50,73 \mathrm{mg} / \mathrm{M} . \mathrm{S}$ pour les protéines. Quant aux teneurs phénoliques, elles sont significativement plus faibles pour 60 jours (20,64 mg/g M.S). Une durée de chauffage de 40 jours, avec un pourcentage de germination de 49,2\%, a entrainé de fortes activités enzymatiques et de fortes teneurs en protéines et en composés phénoliques comparativement à 60 jours $(66,3 \%)$ et 80 jours $(63,73 \%)$. Le métabolisme observé varie selon la durée de chauffage.

(C) 2019 International Formulae Group. All rights reserved.

Mots clés : Traitement, durée de chauffage, polyphénoloxydases, phénylalanine ammonia-lyase, composés phénoliques, protéines.

\section{Effect of heating time of oil palm seeds (Elaeis guineensis Jacq.) On some metabolites during the germination process}

\begin{abstract}
For a good availability of the selected germinated seeds and within a short time, a modified'"' germination by dry heat" technique was initiated. For this purpose the seeds were treated at different heating times. Determinations of polyphenoloxidases (PPO), phenylalanine ammonia-lyase (PAL), total phenolic
\end{abstract}


compounds and proteins were performed for 40, 60 and 80 days at $40 \mathrm{oC}$. The results obtained with albumens are negligible. In embryos, enzymatic activities and protein content decreased with increasing heating time, with respectively 177.7 to $35.07 \mathrm{DO} / \mathrm{min} / \mathrm{g}$ M.S for PPOs, 928.54 to $334 \mathrm{mM}$ cinnamic acid/min/g M.S for PAL and 92.4 to $50.73 \mathrm{mg} / \mathrm{M} . \mathrm{S}$ for proteins. Phenolic levels are significantly lower for 60 days $(20.64 \mathrm{mg} / \mathrm{g}$ M.S.). A 40-day heating period, with a germination rate of $49.2 \%$, resulted in high enzymatic activity and high levels of protein and phenolic compounds compared to 60 days $(66.3 \%)$ and 80 days (63.73\%). The observed metabolism varies according to the duration of heat

(C) 2019 International Formulae Group. All rights reserved.

Keywords: Treatment, heating time, polyphenoloxidases, phenylalanine ammonia-lyase, phenolic compounds, proteins.

\section{INTRODUCTION}

Pour assurer la reproduction de l'espèce, les graines ou semences, organes apparemment inertes, doivent germer, c'est-àdire entrer en activité pour donner une nouvelle plante. Ce processus débute par l'imbibition de la graine qui provoque le développement de l'embryon (Baskin et Baskin, 2004).

Plusieurs techniques sont utilisées pour lever la dormance des graines (Yandou Bio et al., 2015) et accélérer leur germination chez les plantes, surtout celles qui sont cultivées. Ainsi l'effet de la chaleur sur l'amélioration de la capacité de germination des graines a été démontré par Periasamy et al. (2002) et Holou et al. (2011). La germination de graines de certaines espèces du gen re Pinus et Citrus augmentent sensiblement après un trempage dans une solution de cendres ou à une brève exposition des graines à une forte température de 70 à $100{ }^{\circ} \mathrm{C}$ (Herrero et al., 2007).

$\mathrm{La}$ germination des graines s'accompagne d'importants changements biochimiques qui se traduisent par un catabolisme et un anabolisme de composés inhibiteurs ou de substances stimulatrices (Mihoub et al., 2004).

Les composés phénoliques sont impliqués dans de nombreux processus physiologiques comme la croissance cellulaire, la rhizogénèse, la germination des graines ou la maturation des fruits (Lugasi et al., 2003). D'après les travaux de Dicko et al. (2005a) sur le sorgho, les composés phénoliques seraient impliqués dans la germination des graines par le piégeage de l'oxygène de l'air, empêchant ainsi les réactions d'oxydation, ce qui provoque leur entrée en dormance. Au cours de ses réactions de multiples enzymes sont activées, entre autres, la phénylalanine ammonia-lyase (PAL) qui est le précurseur de la synthèse de la plupart des composés phénoliques (Nugroho et al., 2002) et des polyphénoloxydases (PPO) qui oxydent les composés phénoliques facilement en quinones en présence d'oxygène (Concellüm et al., 2004).

$\mathrm{Au}$ moment de la germination, les réserves protéiques sont digérées grâce à des protéases formées par l'embryon (Steber, 2007). Effa et al. (2010) ont montré que les protéines et les polyamines pourraient intervenir au cours des premiers stades de la germination du cola (Cola nitida), à cause du réveil métabolique dû à une plus grande synthèse des enzymes.

L'objectif de ce travail a été de déterminer les activités des PPO, la PAL et la teneur composés phénoliques éthano-solubles ainsi que celle des protéines des graines chauffées à différentes durées au cours du processus de germination

\section{MATÉRIEL ET MÉTHODES \\ Site d'étude}

Les essais de terrain ont été réalisés au CNRA La Mé. Cette station est située sur la route menant à Alépé (latitude $5^{\circ} 22^{\prime} \mathrm{N}-5^{\circ} 24^{\prime} \mathrm{N}$ et longitude $3^{\circ} 47 \mathrm{E}-3^{\circ} \quad 49^{\mathrm{E}}$ ) au Nord-Est d'Abidjan.

\section{Matériel végétal}

Le matériel végétal était composé de graines de palmier à huile (Elaeis guineensis Jacq., variété Dura). Les graines proviennent 
de descendances illégitimes de palmier à huile génotype C70XX. Elles ont été fournies par le Centre National de Recherche Agronomique (CNRA), station La Mé (Côte d'Ivoire). Les analyses ont été menées sur les albumens et les embryons des graines.

\section{Méthodes}

\section{Obtention des graines (étape initiale ou T0)}

Cinq (5) à six (6) mois après la formation des graines (maturité), les régimes de palmier à huile ont été coupés, égrappés et laissés fermenter dans de l'eau pendant une semaine, puis dépulpés. Environ 5400 (cinq mille quatre cents) graines ont été lavées, séchées, puis désinfectées à l'aide d'une préparation fongicide $(5 \mathrm{~g}$ de benlate $+10 \mathrm{~g}$ de dithane M45 dans 10 litres d'eau), pour prévenir le développement de moisissures. Les différentes étapes ci-dessous ont été suivies selon le protocole expérimental décrit par Corrado et Wuidart (1990).

\section{Traitement des graines}

Traitement 1: Trempage et désinfection des graines (T1)

Les graines ont été trempées dans de l'eau de robinet à température ambiante (25$27{ }^{\circ} \mathrm{C}$ ) pendant 7 jours. L'eau de trempage a été renouvelée chaque jour. En fin de trempage, les graines ont été traitées par une solution fongicide comme précédemment pendant 10 à 15 minutes pour éviter le développement de moisissures. Elles sont ensuite étalées sur des sacs de jutes pour leur ressuyage c'est-à-dire l'élimination du surplus d'eau.

Traitement 2 : Chauffage des graines (T2)

Le traitement a consisté d'abord à placer les graines dans des sacs de polyéthylène fermés hermétiquement et ensuite, celles-ci ont été placées dans une salle thermo régulée à $40{ }^{\circ} \mathrm{C} .1800$ graines par traitement (soit 600 graines x 3 répétitions) ont été chauffées pendant 40,60 et 80 jours. La durée de chauffage de 80 jours est celle de référence utilisée actuellement pour la germination des graines des palmiers élites à vulgariser (Corrado et Wuidart, 1990).

Traitement 3 : Retrempage des graines (T3)
Après leur chauffage à $40{ }^{\circ} \mathrm{C}$, les graines ont été retrempées dans de l'eau de robinet à température ambiante $\left(25-27{ }^{\circ} \mathrm{C}\right)$ pendant 5 jours, avec un renouvellement quotidien. En fin de trempage, les graines ont été traitées par un fongicide comme précédemment. Les graines sont alors ressuyées et mises dans de nouveaux sacs.

\section{Isolement des albumens et des embryons des graines}

Après le traitement 3 , deux lots de graines ont été constitués. Le premier lot a servi au prélèvement des albumens et des embryons, le second a servi à faire le test de germination. Pour le premier lot, différents sous-lots ont été constitués selon les différentes durées de chauffage. Au cours de cette expérience, 50 graines pour le stade (T0) et pour le traitement (T1), ainsi que 150 graines pour les traitements $\mathrm{T} 2$ et $\mathrm{T} 3$ ont été prélevées. Les amandes de ces graines des différents lots (T0, T1, T2-40, T2-60, T2-80, T3-40, T3-60 et T3-80) ont été lyophilisées à $60{ }^{\circ} \mathrm{C}$, sous une pression de 8,5 millibars pendant 48 heures. Les embryons ont été retirés des albumens à l'aide de seringues stériles. Pour chaque traitement, deux lots d'échantillons ont été constitués: 1 lot constitué par les albumens et un lot par les embryons. À l'aide d'une broyeuse électrique (KRUPS KM75), les albumens ont été réduits en poudre pour servir aux analyses. Pour chaque analyse, trois répétitions ont été réalisées, à raison de trois essais par répétition, soit neuf essais pour chaque échantillon.

Extraction et dosage des activités enzymatiques

Polyphénoloxydases (PPO)

L'extraction des PPO a été réalisée à basse température $\left(4^{\circ} \mathrm{C}\right)$. Un demi gramme $(0,5 \mathrm{~g})$ d'albumen et $20 \mathrm{mg}$ d'embryons lyophilisés ont été broyées respectivement dans 5 à $2 \mathrm{ml}$ de tampon d'extraction phosphate de sodium $0,1 \mathrm{M} \mathrm{pH} \mathrm{6,5} \mathrm{en}$ présence d'une pincée de sable de fontainebleau.

Le surnageant obtenu après centrifugation a été purifié à l'aide de $0,2 \mathrm{~g}$ 
d'une résine échangeuse d'ions (DOWEX 2) sous agitation au froid pendant $30 \mathrm{~min}$. Le mélange extrait-résine a été centrifugé à 5000 tours $\min ^{-1}$ pendant $20 \mathrm{~min}$. Le surnageant qui en a résulté, a servi au dosage des PPO.

Le dosage de l'activité des polyphénol-oxydases (PPO) a été fait par la méthode de Coseteng et Lee (1987) modifiée et adaptée à notre matériel végétal. Le mélange réactionnel a été composé de $0,2 \mathrm{ml}$ d'extrait enzymatique, $0,3 \mathrm{ml}$ d'une solution de pyrocatéchol $30 \mathrm{mM}$ et d'un (1) $\mathrm{ml}$ d'une solution de tampon phosphate sodium $0,1 \mathrm{M}$ pH 6,5.

Le mélange réactionnel a été incubé pendant $10 \mathrm{~min}$. L'oxydation du pyrocatéchol par les PPO a été mesurée au spectrophotomètre à la longueur d'onde de $500 \mathrm{~nm}$ contre un témoin dans lequel le pyrocatéchol a été remplacé par le tampon phosphate sodium $0,1 \mathrm{M}$ pH 6,5. L'activité des PPO a été exprimée par la variation de la densité optique ( $\Delta$ DO) par minute, par gramme de matière sèche (Ngalani et Crouzet, 1986).

Phénylalanine ammonia-lyase (PAL)

L'extraction de la phénylalanine ammonia-lyase (PAL) a été faite dans les mêmes conditions que celles des PPO, sauf que le tampon d'extraction phosphate de sodium $0,1 \mathrm{M}$ était à $\mathrm{pH} 7,5$. Le surnageant résultant de la centrifugation du broyat a été purifié avec le DOWEX 2 comme précédemment. Le surnageant obtenu, a constitué l'extrait enzymatique.

Le dosage de l'activité de la phénylalanine ammonia-lyase (PAL) a été fait selon la méthode décrite par Zucker (1965) modifiée et adaptée à notre matériel végétal. Le mélange réactionnel a été composé de 0,2 $\mathrm{ml}$ d'extrait enzymatique, d'un (1) $\mathrm{ml}$ d'une solution de phénylalanine $0,1 \mathrm{M}$ et de $0,3 \mathrm{ml}$ d'une solution de tampon phosphate de sodium $0,1 \mathrm{M} \mathrm{pH} 7,5$.

Ce mélange réactionnel a été incubé pendant $20 \mathrm{~min}$ à la température de $25{ }^{\circ} \mathrm{C}$. L'activité de la PAL a été directement proportionnelle à la quantité d'acide cinnamique formée. Cette quantité a été mesurée au spectrophotomètre à la longueur d'onde de $290 \mathrm{~nm}$ contre un témoin dans lequel la phénylalanine a été remplacée par le tampon phosphate de sodium $0,1 \mathrm{M}$ pH 7,5. L'activité de celle-ci a été exprimée en milli moles d'acide cinnamique formé par minute et par gramme de matière sèche (mmol d'acide cinnamique formé/min/g M.S.). Le coefficient d'extinction molaire de l'acide cinnamique est égal à $19600 \mathrm{~cm}^{-1} \mathrm{~mol}^{-1}$.

\section{Extraction et dosage des composés phénoliques}

Les phénols ont été extraits selon la méthode de Sineiro et al. (1996) modifiée et adaptée à notre matériel végétal. $0,5 \mathrm{~g}$ d'albumen et $20 \mathrm{mg}$ d'embryons ont été broyés respectivement dans $10 \mathrm{ml}$ et $3 \mathrm{ml}$ d'éthanol $95 \% \quad(\mathrm{v} / \mathrm{v})$. Le broyat a été centrifugé à 5000 tours $\mathrm{min}^{-1}$ pendant $5 \mathrm{~min}$. Les culots ont été repris respectivement dans 10 et $3 \mathrm{ml}$ d'éthanol $80 \%(\mathrm{v} / \mathrm{v})$ et centrifugé comme précédemment. Les surnageants obtenus après ces deux centrifugations, ajustées à $10 \mathrm{ml}$ et $3 \mathrm{ml}$ avec l'éthanol $50 \%$ $(\mathrm{v} / \mathrm{v})$, ont constitué les extraits bruts qui ont servi au dosage des phénols totaux.

Le dosage des phénols a été fait selon la méthode de Swain et Hillis (1959) modifiée et adaptée à notre matériel végétal. Le mélange réactionnel a été composé, principalement d'acide phosphotungstique et d'acide phosphomolybdique qui ont été réduits en milieu alcalin, parallèlement à l'oxydation des phénols.

Le mélange réactionnel était composé de $0,5 \mathrm{ml}$ de réactif de FolinCiocalteu $1 \mathrm{~N}$, de $1,5 \mathrm{ml}$ de carbonate de sodium $17 \%(\mathrm{p} / \mathrm{v})$ et de 0,5 et $0,1 \mathrm{ml}$ d'extrait brut respectivement d'albumen et d'embryon. Après 45 min d'incubation, l'intensité de la coloration produite par cette réaction, proportionnelle à la concentration des composés phénoliques de l'extrait est suivie au spectrophotomètre à $725 \mathrm{~nm}$.

Pour tenir compte des composés non phénoliques mais pouvant réagir avec le réactif (Folin-Ciocalteu), $0,1 \mathrm{~g}$ de PVP (polyvinylpyrrolidone) a été ajouté à une partie de l'extrait et l'autre ne contenait pas de 
PVP. Les différents mélanges ont été centrifugés à 5000 tours $\min ^{-1}$ pendant $5 \mathrm{~min}$. Le surnageant obtenu sans PVP et celui obtenu avec PVP ont permis de calculer la quantité de composés phénoliques totaux contenus dans les échantillons analysés.

Les quantités de phénols totaux ont été déterminées à l'aide d'une droite d'étalonnage réalisée avec une solution de tyrosine $(100 \mu \mathrm{g} / \mathrm{ml})$. L'équation de droite avec la tyrosine a été de $\mathrm{Y}=0,021 \mathrm{x}$ $+0,053 \mathrm{y}, \quad \mathrm{R} 2=0,999$ avec comme caractéristiques, y est l'absorbance et $\mathrm{x}$ les concentrations de tyrosine en $\mathrm{mg} / \mathrm{ml}$. NB : R2 ; c'est le $\mathrm{R}$ au carrée. Ces valeurs ont été estimées en milligrammes de phénols par gramme de matière sèche $(\mathrm{mg}$ de phénols / $\mathrm{g}$ M.S).

\section{Extraction et dosage des protéines}

L'extraction des protéines a été réalisée à basse température $\left(4{ }^{\circ} \mathrm{C}\right)$ par broyage de 0,5 g d'albumen ou $20 \mathrm{mg}$ d'embryons lyophilisés en présence d'une pincée de sable de fontainebleau. Le broyage a été fait respectivement dans 5 et $2 \mathrm{ml}$ de tampon phosphate de sodium $0,1 \mathrm{M} \mathrm{pH} 7,5$ en présence de $0,1 \mathrm{~g}$ de polyvinylpyrrolidone (PVP). Le milieu d'extraction contenait du polyéthylène glycol (PEG 6000) $(0,5 \%, \mathrm{p} / \mathrm{v})$ pour fixer les composés phénoliques, un réducteur (mercaptoéthanol) $15 \mathrm{mM}(\mathrm{v} / \mathrm{v})$ dans le but d'éviter des oxydations enzymatiques, un complexant (EDTA) $10 \mathrm{mM}$ $(\mathrm{p} / \mathrm{v})$ afin de réduire l'inhibition des ions métalliques. La protection des sites enzymatiques a été obtenue par addition de thiosulfate de sodium $(0,5 \%, \mathrm{p} / \mathrm{v})$ et de glycérol $(15 \%, v / v)$ au milieu d'extraction.

Le broyat a été centrifugé à 15000 tours $\min ^{-1}$ pendant $20 \mathrm{~min}$, à $4{ }^{\circ} \mathrm{C}$. Le surnageant obtenu a constitué l'extrait brut. Le dosage des protéines a été fait selon la méthode de Bradford (1976) où l'on a mesuré au spectrophotomètre à la longueur d'onde de $595 \mathrm{~nm}$, la densité optique du complexe coloré des protéines avec le bleu de Coomassie. Le mélange réactionnel a été composé de $0,1 \mathrm{ml}$ d'extrait brut, $0,9 \mathrm{ml}$ d'une solution de tampon phosphate de sodium $0,1 \mathrm{M}$ pH 7,5 et $2 \mathrm{ml}$ de solution de bleu de coomassie (réactif).

Un témoin a été réalisé avec le tampon phosphate de sodium $0,1 \mathrm{M} \mathrm{pH}$ 7,5. La réaction a eu lieu à l'obscurité, et les tubes à essais ont été placés dans un bain de glace, pendant $30 \mathrm{~min}$. Une solution de Sérum Albumine Bovine $(\mathrm{SAB})$ à $50 \mu \mathrm{g} / \mathrm{ml}$ a été utilisée comme solution protéinique de référence. Le taux de protéines a été exprimé en milligrammes par gramme de matière sèche $(\mathrm{mg} / \mathrm{g} \mathrm{MS})$, déterminé à l'aide d'une courbe étalon.

\section{Germination des graines}

Les sacs contenant les graines ont été transférés dans une salle à la température ambiante $\left(25-27^{\circ} \mathrm{C}\right)$ puis la germination a été suivie pendant 8 semaines.

\section{Pourcentage de germination}

Le pourcentage de germination des graines $(\% \mathrm{G})$ a été évalué après 8 semaines d'incubation par le comptage des graines ayant germées. Le pourcentage de germination est calculé selon la formule ;

$$
\% \mathbf{G}=\text { (N.G.G. / N.T.G.) } \times 100
$$

$\% \mathrm{G}=$ pourcentage de germination

N.G.G. = nombre de graines ayant germées

N.T.G. $=$ nombre total de graines mises à germer

\section{Analyses statistiques des données}

Les analyses statistiques des résultats ont été faites avec le logiciel Statistica 6,0 par la procédure ANOVA. Une analyse de variance, suivie de la comparaison de moyenne \pm écart type par la méthode de Newmann-Keuls au seuil de $5 \%$, a été effectuée.

\section{RÉSULTATS}

\section{Activités des phénol-oxydases (PPO)}

L'on a également noté une différence significative entre les embryons et les albumens quel que soit le traitement. Au stade initial (T0), l'activité des PPO des embryons a été de $138,11 \mathrm{DO} / \mathrm{min} / \mathrm{g}$ M.S, et de 6,24 DO/min/g M.S pour les albumens. 
Pour l'étape du trempage (T1), l'activité est passée à $76,43 \mathrm{DO} / \mathrm{min} / \mathrm{g}$ M.S et 7,53 $\mathrm{DO} / \mathrm{min} / \mathrm{g}$ M.S respectivement pour les embryons et les albumens. Cette teneur a varié de 35,07 à $177,7 \mathrm{DO} / \mathrm{min} / \mathrm{g}$ M.S, pour une durée de chauffage (T2) allant de 40 jours à 80 jours pour les embryons; et de 3,55 à 6,14 $\mathrm{DO} / \mathrm{min} / \mathrm{g}$ M.S pour les albumens dans les mêmes conditions de chauffage. Au cours du retrempage (T3) les valeurs des teneurs ont varié de 63,6 à $259,9 \mathrm{DO} / \mathrm{min} / \mathrm{g}$ M.S et de 2,74 à 4,5 DO/min/g M.S respectivement chez les embryons et les albumens (Tableau 1).

\section{Activités de la phénylalanine ammonia- lyase (PAL)}

Une différence significative entre les embryons et les albumens a été relevée quel que soit le traitement. Au stade initial (T0), l'activité de la PAL des embryons a été de $252,7 \mathrm{mM}$ d'acide cinnamique $/ \mathrm{min} / \mathrm{g}$ M.S et de 24,43 mM d'acide cinnamique/ min/g M.S pour les albumens. Pour l'étape du trempage (T1), l'activité est passée à 75,6 $\mathrm{mM}$ d'acide cinnamique $/ \mathrm{min} / \mathrm{g}$ M.S. et $5,07 \mathrm{mM}$ d'acide cinnamique/min/g M.S respectivement pour les embryons et les albumens. Cette activité a varié de 334,0 à 928,54 mM d'acide cinnamique/min/g M.S., pour une durée de chauffage (T2) allant de 40 jours à 80 jours pour les embryons; et de 18,98 à $56,24 \mathrm{mM}$ d'acide cinnamique $/ \mathrm{min} / \mathrm{g}$ M.S pour les albumens dans les mêmes conditions de chauffage. Au cours du retrempage (T3) les valeurs des activités ont varié de 154,0 à $181,57 \mathrm{mM}$ d'acide cinnamique $/ \mathrm{min} / \mathrm{g}$ M.S et de 26,74 à $34,41 \quad \mathrm{mM}$ d'acide cinnamique/min/g M.S respectivement chez les embryons et les albumens (Tableau 2).

\section{Teneur en phénols totaux}

Les analyses des résultats ont également montré une différence significative entre les embryons et les albumens quel que soit le traitement. Au stade initial (T0), la teneur en composés phénoliques des embryons a été de $10,17 \mathrm{mg} / \mathrm{g}$ M.S et de 0,69 $\mathrm{mg} / \mathrm{g}$ M.S pour les albumens. Pour l'étape du trempage (T1), ce taux est passé à $18,1 \mathrm{mg} / \mathrm{g}$ M.S et $0,17 \mathrm{mg} / \mathrm{g}$ M.S respectivement pour les embryons et les albumens. Cette teneur a varié de 20,64 à $28,85 \mathrm{mg} / \mathrm{g}$ M.S, pour une durée de chauffage (T2) allant de 40 jours à 80 jours pour les embryons et de 0,08 à $0,85 \mathrm{mg} / \mathrm{g}$ M.S pour les albumens dans les mêmes conditions de chauffage. Au cours du retrempage (T3) les valeurs des teneurs ont varié de 7,69 à 39,46 $\mathrm{mg} / \mathrm{g}$ M.S et de 0,25 à $3,6 \mathrm{mg} / \mathrm{g}$ M.S respectivement chez les embryons et les albumens (Tableau 3).

\section{Teneur en protéines}

L'analyse statistique a montré une différence significative entre les embryons et les albumens quel que soit le traitement. Au stade initial (T0), la teneur en protéines des embryons a été de 39,08 $\mathrm{mg} / \mathrm{g}$ M.S et de 1,35 mg/g M.S pour les albumens. Pour l'étape du trempage (T1), ces taux sont passés à 35,01 mg/g M.S et 3,87 mg/g M.S respectivement pour les embryons et les albumens. Cette teneur a baissé de 92,4 à $50,73 \mathrm{mg} / \mathrm{g}$ M.S, pour une durée de chauffage (T2) allant de 40 jours à 80 jours pour les embryons; et varié de 3,9 à 5,84 mg/g M.S pour les albumens dans les mêmes conditions. Au cours du retrempage (T3) les valeurs des teneurs ont varié de 50,9 à 59,76 $\mathrm{mg} / \mathrm{g}$ M.S et de 1,74 à $3,97 \mathrm{mg} / \mathrm{g}$ M.S respectivement chez les embryons et les albumens (Tableau 4).

\section{Pourcentage de germination}

Le Tableau 5 montre que le pourcentage de germination des graines est statistiquement semblable pendant 40, 60 et 80 jours de chauffage. Cependant le pourcentage de germination le plus élevé $(66,3 \%)$ a été obtenu pour 60 jours de chauffage suivi de 80 jours $(63,73 \%)$ et enfin de 40 jours $(49,2 \%)$. 
Tableau 1 : Évolution de l'activité des phénol-oxydases (PPO) (DO/min/g M.S) dans des graines de palmier à huile au cours du processus de germination.

\begin{tabular}{|c|c|c|c|c|c|c|}
\hline \multicolumn{7}{|c|}{ Activité des polyphénol-oxydases (DO/min/g M.S) } \\
\hline Traitements & $\begin{array}{l}\text { Durée } \\
\text { (jours) }\end{array}$ & chauffage & Embryons & Albumens & $\mathbf{F}$ & $\mathbf{P}$ \\
\hline $\begin{array}{l}\text { Etape initiale } \\
\text { (T0) }\end{array}$ & & 0 & $138,11 \pm 6,88^{\mathrm{a}}$ & $6,24 \pm 0,25^{\mathrm{b}}$ & 1462,2 & 0,000000 \\
\hline $\begin{array}{l}\text { Trempage de } 7 \\
\text { jours (T1) }\end{array}$ & & 0 & $76,43 \pm 2,08^{\mathrm{a}}$ & $7,53 \pm 0,09^{b}$ & 1097,7 & 0,000000 \\
\hline \multirow[t]{3}{*}{ Chauffage (T2) } & & $\mathrm{T} 2-40$ & $177,7 \pm 8,4^{\mathrm{a}}$ & $6,14 \pm 0,39^{d}$ & \multirow[t]{3}{*}{163,9} & \multirow[t]{3}{*}{0,0019} \\
\hline & & $\mathrm{T} 2-60$ & $110,08 \pm 4,44^{\mathrm{b}}$ & $3,55 \pm 0,3^{\mathrm{d}}$ & & \\
\hline & & $\mathrm{T} 2-80$ & $35,07 \pm 0,99^{\mathrm{c}}$ & $4,9 \pm 0,16^{\mathrm{d}}$ & & \\
\hline \multirow{3}{*}{$\begin{array}{l}\text { Retrempage de } 5 \\
\text { jours (T3) }\end{array}$} & & T3-40 & $259,9 \pm 20,79^{a}$ & $4,5 \pm 0,59^{\mathrm{d}}$ & \multirow[t]{3}{*}{73,3} & \multirow[t]{3}{*}{0,000008} \\
\hline & & T3-60 & $92,97 \pm 2,54^{\mathrm{b}}$ & $3,04 \pm 0,13^{\mathrm{d}}$ & & \\
\hline & & T3-80 & $63,6 \pm 3,4^{c}$ & $2,74 \pm 0,14^{\mathrm{d}}$ & & \\
\hline
\end{tabular}

Tableau 2: Évolution de l'activité de la phénylalanine ammonia-lyase (PAL) (mM d'acide cinnamique/min/g M.S) dans des graines de palmier à huile au cours du processus de germination.

\section{Activité de la phénylalanine ammonia-lyase (Mm d'acide cinnamique/min/g M.S)}

\begin{tabular}{llllll}
\hline Traitements & $\begin{array}{l}\text { Durée chauffage } \\
\text { (jours) }\end{array}$ & Embryons & Albumens & F & P
\end{tabular}

\begin{tabular}{|c|c|c|c|c|c|}
\hline $\begin{array}{l}\text { Etape initiale } \\
\text { (T0) }\end{array}$ & 0 & $252,7 \pm 7,04^{\mathrm{a}}$ & $24,43 \pm 0,56^{b}$ & 1045,5 & 0,000000 \\
\hline $\begin{array}{l}\text { Trempage de } 7 \\
\text { jours (T1) }\end{array}$ & 0 & $75,6 \pm 1,4^{\mathrm{a}}$ & $5,07 \pm 0,34^{b}$ & 2439,5 & 0,000000 \\
\hline \multirow[t]{3}{*}{ Chauffage (T2) } & $\mathrm{T} 2-40$ & $928,54 \pm 27,4^{\mathrm{a}}$ & $24,66 \pm 1,8^{\mathrm{e}}$ & \multirow[t]{3}{*}{152,58} & \multirow[t]{3}{*}{0,000000} \\
\hline & $\mathrm{T} 2-60$ & $413,34 \pm 30,6^{b}$ & $18,98 \pm 0,56^{\mathrm{f}}$ & & \\
\hline & $\mathrm{T} 2-80$ & $334,00 \pm 21,4^{\mathrm{c}}$ & $56,24 \pm 5,64^{\mathrm{d}}$ & & \\
\hline \multirow{3}{*}{$\begin{array}{l}\text { Retrempage de } 5 \\
\text { jours (T3) }\end{array}$} & T3-40 & $154,00 \pm 9,55^{\mathrm{b}}$ & $26,74 \pm 2,78^{\mathrm{d}}$ & \multirow[t]{3}{*}{73,3} & \multirow[t]{3}{*}{0,024} \\
\hline & T3-60 & $154,83 \pm 6,11^{b}$ & $34,41 \pm 2,42^{\mathrm{c}}$ & & \\
\hline & T3-80 & $181,57 \pm 6,4^{\mathrm{a}}$ & $26,9 \pm 2,47^{\mathrm{d}}$ & & \\
\hline
\end{tabular}


Tableau 3 : Évolution des teneurs en phénols totaux (mg/g M.S) dans les graines de palmier à huile au cours du processus de germination.

\begin{tabular}{|c|c|c|c|c|c|}
\hline \multicolumn{6}{|c|}{ Teneurs en phénols totaux (mg/g M.S) } \\
\hline Traitements & Durée chauffage (jours) & Embryons & Albumens & $\mathbf{F}$ & $\mathbf{P}$ \\
\hline $\begin{array}{ll}\text { Etape } & \text { initiale } \\
\text { (T0) } & \end{array}$ & 0 & $10,17 \pm 0,57^{\mathrm{a}}$ & $0,69 \pm 0,04^{\mathrm{b}}$ & 274,47 & 0,000000 \\
\hline $\begin{array}{lll}\text { Trempage } & \text { de } & 7 \\
\text { jours (T1) } & & \end{array}$ & 0 & $18,1 \pm 0,77^{\mathrm{a}}$ & $0,17 \pm 0,01^{b}$ & 537,56 & 0,000000 \\
\hline \multirow[t]{3}{*}{ Chauffage (T2) } & $\mathrm{T} 2-40$ & $26,14 \pm 1,38^{\mathrm{a}}$ & $0,08 \pm 0,00^{\mathrm{c}}$ & 11,04 & 0,0019 \\
\hline & T2-60 & $20,64 \pm 0,77^{\mathrm{b}}$ & $0,85 \pm 0,06^{\mathrm{c}}$ & & \\
\hline & $\mathrm{T} 2-80$ & $28,85 \pm 1,65^{\mathrm{a}}$ & $0,61 \pm 0,02^{\mathrm{c}}$ & & \\
\hline \multirow{3}{*}{$\begin{array}{l}\text { Retrempage de } 5 \\
\text { jours (T3) }\end{array}$} & T3-40 & $7,69 \pm 0,54^{b}$ & $0,25 \pm 0,02^{\mathrm{c}}$ & 36,2 & 0,000008 \\
\hline & T3-60 & $39,46 \pm 4,00^{\mathrm{a}}$ & $3,6 \pm 0,26^{\mathrm{c}}$ & & \\
\hline & T3-80 & $35,22 \pm 2,14^{\mathrm{a}}$ & $3,04 \pm 0,4^{\mathrm{c}}$ & & \\
\hline
\end{tabular}

Tableau 4 : Évolution des teneurs en protéines (mg/g M.S) dans les graines de palmier à huile au cours du processus de germination.

\begin{tabular}{|c|c|c|c|c|c|}
\hline \multicolumn{6}{|c|}{ Teneurs en protéines (mg/g M.S) } \\
\hline Traitements & Durée chauffage (jours) & Embryons & Albumens & $\mathbf{F}$ & $\mathbf{P}$ \\
\hline $\begin{array}{l}\text { Etape initiale } \\
\text { (T0) }\end{array}$ & 0 & $39,08 \pm 1,8^{\mathrm{a}}$ & $\begin{array}{l}1,35 \pm 0,14 \\
\text { b }\end{array}$ & 438,5 & 0,000000 \\
\hline $\begin{array}{lll}\text { Trempage } & \text { de } & \\
\text { jours (T1) } & & \end{array}$ & 0 & $35,01 \pm 1,03^{\mathrm{a}}$ & $3,87 \pm 0,14^{b}$ & 892,16 & 0,000000 \\
\hline \multirow[t]{3}{*}{ Chauffage (T2) } & $\mathrm{T} 2-40$ & $92,4 \pm 3,9^{\mathrm{a}}$ & $5,84 \pm 0,17^{\mathrm{d}}$ & \multirow[t]{3}{*}{38,76} & \multirow[t]{3}{*}{0,000006} \\
\hline & T2-60 & $61,67 \pm 3,4^{b}$ & $3,9 \pm 0,3^{\mathrm{d}}$ & & \\
\hline & $\mathrm{T} 2-80$ & $50,73 \pm 2,7^{\mathrm{c}}$ & $5,07 \pm 0,4^{\mathrm{d}}$ & & \\
\hline \multirow{3}{*}{$\begin{array}{l}\text { Retrempage de } 5 \\
\text { jours (T3) }\end{array}$} & T3-40 & $50,90 \pm 5,14^{\mathrm{a}}$ & $3,96 \pm 0,4^{b}$ & \multirow[t]{3}{*}{3,05} & \multirow[t]{3}{*}{0,08} \\
\hline & T3-60 & $59,76 \pm 2,24^{\mathrm{a}}$ & $1,74 \pm 0,06^{\mathrm{b}}$ & & \\
\hline & T3-80 & $58,9 \pm 1,47^{\mathrm{a}}$ & $3,04 \pm 0,4^{b}$ & & \\
\hline
\end{tabular}

Les différents stades et traitements T0 et T1; T2-40; T2-60; T2-80; T3-40; T3-60; T3-80; 40, 60 et 80 jours durées de chauffage à ces stades. Chaque stade ou traitement représente la moyenne de trois (3) expériences distinctes (chaque expérience comporte 3 essais). Pour un même traitement, les moyennes suivies de la même lettre ne sont pas significatifs au seuil de $5 \%$ selon le test de Newmann-Keuls.

Tableau 5 : Pourcentage de germination de graines de palmier à huile.

\begin{tabular}{cc}
\hline Durée de chauffage (jours) & Pourcentage de germination (\% G) \\
\hline 40 & $49,2 \pm 6,04^{\text {a }}$ \\
\hline 60 & $66,3 \pm 0,75^{\text {a }}$ \\
\hline 80 & $63,73 \pm 10,15^{\text {a }}$ \\
\hline $\mathrm{F}$ & 2,057 \\
\hline $\mathrm{P}$ & 0,209
\end{tabular}

Les chiffres suivis de la même lettre ne sont pas significativement différents au seuil de 5\% selon le test de Newmann-Keuls. 


\section{DISCUSSION}

\section{Les PPO, la PAL et les composés phénoliques}

Le processus de germination débute par la phase d'imbibition qui permet l'hydratation des tissus. L'action directe de l'eau va activer les réserves oléagineuses. Une partie de ses dernières va servir directement aux oxydations respiratoires et la plus grande partie est convertie en glucides (Shockey et al., 2002). Une activité catabolique est mise en place et permet à l'embryon de se développer à partir de l'énergie provenant de la dégradation des réserves accumulées dans l'albumen. Nos résultats traduisent effectivement le processus de germination initié. Ainsi, au niveau de l'albumen, les composés phénoliques significativement faibles baissent au profit de l'embryon au cours des différentes durées de chauffage. Cette situation pourrait s'expliquer par l'oxydation de ces composés et limiterait la respiration au cours de notre étude. Lorsqu'une graine est imbibée, l'oxygène doit traverser les enveloppes en se dissolvant dans l'eau d'imbibition pour alimenter l'embryon en oxygène Dicko et al. (2006a). Cependant, la présence fréquente de composés phénoliques dans les enveloppes diminuerait la quantité d'oxygène disponible pour l'embryon. En effet, ces composés qui se dissolvent dans l'eau d'imbibition se comportent comme un véritable piège à oxygène car ils s'oxydent en présence de ce gaz sous l'action de polyphénol-oxydases (Bradford et al., 2007).

La quantité de phénols totaux dans les embryons a été opposée à celle de l'activité des polyphénoloxydases (PPO), tandis qu'au niveau des albumens ces paramètres restent faibles. En effet, quand l'activité des PPO a été forte la quantité de phénols totaux a été faible au stade (T2-40). Tandis qu'aux stades (T2-60 et T2-80), quand l'activité des PPO a été faible, la quantité de phénols totaux a été élevée, ce qui montre bien que les PPO dégraderaient les phénols (Dogbo et al., 2008). Ces produits d'oxydation, tels que les o-quinones, réagiraient avec les acides aminés et les protéines nécessaires à la mise en place de nouveaux tissus (Concellüm et al., 2004).

Les variations de l'activité de la PAL seraient également liées aux teneurs en phénols totaux. La forte activité de la PAL pour 40 jours de chauffage aurait permis la synthèse de certains phénols inhibiteurs de la germination (Nugroho et al., 2002). La biosynthèse des composés phénoliques se faisant par la PAL, l'augmentation de l'activité de celle-ci devrait entrainer l'élévation des composés phénoliques. Nos résultats sont contraires à ceux de Dogbo et al. (2008). Ces résultats pourraient s'expliquer par le fait que la voie de synthèse des composés phénoliques emprunterait une autre voie que celle de l'acide cinnamique.

\section{Protéines}

L'augmentation des protéines dans les embryons au cours des traitements proviendrait de la synthèse de nouvelles protéines qui seront utilisées par la suite pour la croissance de la plantule (Koornnef et al., 2002). La durée de chauffage de 40 jours ayant présenté une faible teneur en protéines au moment du retrempage a occasionné une faible germination. Ce qui n'a pas été le cas pour 60 et 80 jours dans les mêmes conditions. La teneur en protéines dans les albumens, bien qu'augmentant au cours des différents stades, a été très faible comparativement à celle des embryons (Oracz et al., 2007). La quantité élevée des protéines dans les embryons pourrait s'expliquer par la synthèse de protéines de structures et enzymatiques. Les travaux effectués par Effa et al. (2010), ont montré qu'au cours de la germination des graines de cola (Cola nitida), les acides aminés ont augmenté, traduisant leur forte mobilisation au cours de ces phénomènes. Pour Aphalo et al. (2009), ces protéines interviendraient dans la mise en place de nouveaux tissus. Cependant, la durée de chauffage est déterminante. Ainsi, la durée de chauffage de 60 jours a été favorable à une bonne germination dans notre cas. 


\section{Pourcentage de germination}

Pour une longue durée de chauffage, le pourcentage de germination des graines devient élevé. Il semblerait que l'application d'une chaleur pendant au moins 40 jours favorise la présence de substances biochimiques ou physiologiques en quantité suffisante pour provoquer la germination des graines (Duval et Shetty, 2001; Mihoub et al., 2004).

La température joue un rôle important dans la germination des graines comme l'ont montré Mounkaila et al. (2015). Dans le cadre précis de notre travail, les graines sont placées pendant un temps long à une température douce de $40{ }^{\circ} \mathrm{C}$ pour provoquer la dégradation des substances inhibitrices de la germination (Fondom et al., 2010). Le chauffage des graines pendant 60 jours a donné un important pourcentage de germination par rapport à 80 jours utilisés au CNRA (Beugré et al., 2009).

Le chauffage des graines, combinées à leur trempage dans de l'eau de robinet pendant le processus de germination, permettrait le réveil métabolique de celles-ci pour provoquer l'allongement de l'embryon (Herrero et al., 2007).

\section{Conclusion}

L'objectif de cette étude était d'évaluer l'effet de la durée de chauffage sur quelques métabolites au cours du processus de germination des graines. Au niveau des embryons, la durée de chauffage des graines pendant 60 jours a entrainé une faible quantité de composés phénoliques, une relative augmentation de protéines et une activité modérée de la PAL et des PPO. Les graines chauffées pendant 40 jours ont eu une teneur de composés phénoliques relativement élevée, une teneur importante de protéines et une activité très forte des enzymes étudiées. Quant à la durée de 80 jours (témoin), elle a entrainé une quantité élevée de composés phénoliques, une relative augmentation des protéines et une faible activité des enzymes comparativement aux autres durées de chauffage. Au niveau de l'albumen, ces paramètres sont restés significativement faibles.
Tous ces résultats montrent qu'au cours de la germination des graines oléagineuses, les composés de réserves nouvellement formés quittent l'albumen pour l'embryon. La Pal précurseur de la synthèse des composés phénoliques, serait activée. Sa présence stimulerait dans l'embryon la production de composés phénoliques. Ces derniers vont s'oxyder en quinones en présence d'oxygène sous l'action des PPO, ce qui induirait la production d'énergie provoquant la germination. Pour mieux maitriser la signification physiologique de la germination des graines oléagineuses, nous envisageons d'étendre nos travaux sur l'activité de la TAL (tyrosine ammonia-lyase).

\section{CONFLIT D'INTERETS}

Aucun conflit d'intérêts potentiel n'a été signalé par les auteurs.

\section{CONTRIBUTIONS DES AUTEURS}

MMB a réalisé les manipulations, analysé les données et rédigé l'article. JYK et EKK ont supervisé l'étude et la rédaction scientifique. FKYK et SESY ont contribué à la correction du manuscrit.

\section{REMERCIEMENTS}

Les auteurs remercient le Centre National de Recherche Agronomique (CNRA) station de La Mé (Côte d'Ivoire), pour la fourniture des graines du palmier à huile.

\section{REFERENCES}

Aphalo P, Martinez EN, Anon MC. 2009. Structural modifications of Amaranth proteins during germination. Journal of Protein Chemistry, 28: 131-138. DOI: 10.1007/s10930-009-9173-4

Baskin JM, Baskin CC. 2004. A classification system for seed for dormancy, Seed Science Research, 14: 1-16. DOI: https://doi.org/10.1079/SSR2003150

Beugré MM, Kouakou KL, Bognonkpé JP, Konan KE, Kouakou TH, Kouadio J. 2009. Effect of storage and heat treatment on the germination of oil palm (Elaeis guineensis Jacq.) seed. African Journal of Agricultural Research, 4 (10): 931-937. 
Bradford MM. 1976. A rapid and sensitive method for the quantification of microgram quantities of protein utilizing the principe of protein-dye binding. Analogy Biochemistry, 72: 248-254.

Bradford KJ, Côme D, Corbineau F. 2007. Quantifying the oxygen sensitivity of seed germination using a population-based threshold model. Seed Science Research, 17(1): 33-43. DOI: https://doi.org/10.1017/S09602585076573 89.

Concellüm A, Aŏun Mc, Chaves AR. 2004. Characterizationand change in polyphenoloxidase from eggplant (Solanum melongena L.) during storage at low temperature. Food Chemistry, 88: 1724. DOI https://doi./10.1016/j.foodchem.2004.017.

Corrado F, Wuidart W. 1990. Ger mination des graines de palmier à huile (E. guineensis) en sacs de polyéthylène: Méthode par «chaleur sèche». Oléagineux, $\mathbf{4 5}(11)$ : 511-514.

Coseteng MY, Lee CY. 1987. Changes in apple plyphenol oxidase and polyphenol concentration in relation to degree of browning, Journal Food Science, 52: 985989.

Dicko MH, Gruppen H, Zouzouho OC, Traoré AS, van Berkel WJH, Voragen AGH. 2006a. Effects of germination on the activities of amylase and phenolic enzymes in sorghum varieties grouped according to food end-use properties. Journal Science Food Agricultural, 86: 953-963.

DOI: https://doi.org/10.1002/jsfa.2443.

Dogbo DO, Bekro MJA, Bekro YA, Sie RS, Gogbeu SJ, Traoré A. 2008. Influence de l'acide salicylique sur la synthèse de la phényalanine ammonia-lyase, des polyphénoloxydases et l'accumulation des composés phénoliques chez le manioc (Manihot esculenta Crantz). Sciences et Nature, $\quad 5$ (1): $1-13 . \quad$ DOI: http://dx.doi.org/10.4314/scinat.v5i1.4214 7

Duval B, Shetty K. 2007. The stimulation of phenolics and antioxidant in Pea (Pisum sativum) elicited by genetically transformed anise root extract. Journal of Food Biochemical, 25: 361-377. DOI: https://doi.org/10.1111/j.1745-

4514.2001.tb00746.x

Effa OP, Niemenak N, Omokol ND, Lieberei R. 2010. Change in amino acids content during germination and seedling growth of Cola sp African. Journal of Biotechnology, 9(35): 5632-5642. http://www.academicjournals.org/AJB.

Fondom NY, Etta CE, Mih AM. 2010. Breaking seed dormancy, revisiting heat-treatment duration on germination and subsequent seedling growth of oil palm (Elaeis guineensis Jacq.) progenies. Journal of Agricultural Science, 2(2): 101-110. DOI: 10.5539/jas.v2n2p101.

Herrero C, San Martin R, Bravo F. 2007. Effect of heat and ash treatments on germination of Pinus pinaster and Cistus laurifolius. Journal of Arid Environnement, 70 : 540548.

DOI: https://doi.org/10.1016/j.jaridenv.2006.12. 027.

Holou YAR, Teka SO, Kindomihou MV, Sinsin AB. 2011. Germination et utilization de Ceasalpinia benthamiana (Baillon) P.S. Herendeen et J.L. Zarucchi (LeguminosaeCaesalpiniacee) dans l'aménagement antiérosif des retenues d'hydraulique pastorale au Bénin. Int. J. Biol. Chem. Sci., 5(2): 533-545. DOI: http://dx.doi.org/10.4314/ijbcs.v5i2.72100.

Koornef M, Bentsink L, Bentsink L, Hilhorst H. 2002. Seed dormancy and germination. Plant Biology, 5: 33-36. DOI: 10.1016/s1369-5266(01)00219-9.

Lugasi A, Hovari J, Sagi KV, Biro L. 2003. The role of antioxidant phytonutrients in the prevention of diseases. Acta Biologica Szegedientsis, (1-4): 119-125.

Mihoub A, Chaoui A, El Ferjani E. 2004. Changements biochimiques par le cadmium et le cuivre au cours de la germination des graines de petit pois (Pisum sativum L.). Comptes Rendus Biologies, 328: 33-41. DOI: https://doi.org/10.1016/j.crvi.2004.10.003. 
Moukaila S, Soukeraji B, Abdoulaye A, Mahamane A, Ikhiri K, Morou B, Karim S. 2015. Essai de germination et croissance de Atertemisia anna $\mathrm{L}$ au Niger. Int. J. Biol. Chem. Sci., 9(1): 108120.

DOI: http://dx.doi.org/10.4314/ijbcs.v9i1.11.

Ngalani J, Crouzet J. 1986. Mise en évidence et étude de quelques propriétés de la phenoloxydase (PPO) de la banane plantain. Revue Scientifique et Technique des Services Scientifiques et Agricoles, 2 : 35-45.

Nugroho LH, Verberne MC, Verpoorte R. 2002. Activities of enzymes involved in phenylpropanoid pathway in constituvelly salicylic acid-producing tobacco plants. Plant Physiology Biochemistry, 40: 755760. DOI: 10.1016/S0981-9428(02)014377.

Oracz K, Bouteau HEM, Farrant JM, Cooper K, Belghazi M, Job C, Job D, Corbineau F, Bailly C. 2007. ROS production and protein oxidation as a novel mechanism for seed dormancy alleviation. Plant Journal, 50: 452-465. DOI: https://doi.org/10.1111/j.1365313X.2007.03063.X

Periasamy A, Gopapal K, Soh AC. 2002. Productivity improvements in seed processing techniques for commercial oil palm seed production. The Planter, 78, 429-442.
Shockey JM, Fulda MS, Browse JA. 2002. Arabidopsis contains nine long-chainacylcoenzymeA synthetase genes that participate in fatty acid and glycerolipid metabolism. Plant Physiology, 129: 17101722. DOI

Sineiro J, Dominguez H, Nunez MJ, Lema JM. 1996. Ethanol extraction of polyphenols in a immersion extractor, Effect of pulsing flow. Journal of America Oil Chemists Society, 73 (9): 1121-1125.

Steber CM. 2007. De - repression of seed germination by GA signaling. In: Bradford K. and Nonogaki H. (Eds). Seed development, dormancy and germination. Annual Plant Reviews, 27: 248-263. DOI: 10.1002/9780470988848.ch10

Swain T, Hillis WE. 1959. Phenolic constituents of Punus domestica, the quantitative analyse of phenol constituents. Journal Science Food Agricol., 10: 63-68. DOI: https://dx.doi.org/10.1002/jsfa.274010011 0

Yandou Bio I, Soumana I, Rabiou $\mathrm{H}$ et Mahamane A. 2019. Effets des traitements sur la germination de Acacia tortilis subsp. raddiam (Savi) Brenan au Niger, Sahel. Int. J. Bio. Chem. Sci., 13 (2): 776-790. DOI: http://dx.org/104314/ijbcs.v13i2.16.

Zucker M. 1965. Induction of phenylalanine deaminase by light and its relation to Chlorogenic acid synthesis in Potato tuber tissues. Plant Physiology, 40 : 779-784. DOI : 10.1104/pp.40.5.779. 